\title{
COMBAT VEHICLE RELIABILITY ASSESSMENT SIMULATION MODEL (CVRASM)
}

\author{
Stephen R. Parker \\ Department of Decision Sciences \\ U.S. Army Logistics Management College \\ Fort Lee, Virginia 23801-6050
}

\begin{abstract}
A simulation model capable of analyzing manufacturers' claims of reliability has been developed. The model takes into account the manufacturers' predetermined reliability inputs and incorporates these statements into the army's current maintenance and repair system. The model portrays the disposition or status of vehicles over time. This information may be used to initialize other combat models in which reliability may not previously have been a consideration.

The program is formulated with a FORTRAN user interface to allow the modeler to provide the initial data base for the particular vehicle types and maintenance facilities. Additionally, the model portrays the commander's predefined combat minimum and "flags" such information to the commander that his unit is combat ineffective due to down time in repairs. Sensitivity analysis is easily accomplished through the input system data base.
\end{abstract}

\section{INTRODUCTION}

A simulation model depicting reliability of systems and subsystems of combat vehicles has been further developed. In particular, it is necessary to evaluate systems which the army purchases from contracts and evaluate these systems based upon the manufacturers' reliability specifications. Such comparisons provide the important information as to the expected levels which can be assumed to exist prior to and during combat. Combining this information is very critical to major commands especially in operations such as Desert Storm where the operating environment had been critically changed and must allow for the desert operating characteristics. For example, in the case of vehicles in operation in the gulf theater, air cleaners had been consistently in short supply causing major down time for cleaning and repairing of systems. Another example was the critical shortage of helicopter blades for the exchange of blades quickly diminished during desert flight operations.

The evaluation of reliability is not new. Previous studies have led to the development of mainframe versions of reliability analysis. One such model, COVERS, (Combat Vehicle RAM Simulation) was developed for use in determining reliability, availability, and maintainability (RAM) requirements for new vehicles. Such models were primarily concerned with the effects of various changes in support policy and logistics concepts on the operational availability of equipment. The primary problem with this type of simulation model is the inflexibility to modify existing code to a rapidly changing environment. The COVERS model, as with many other earlier reliability models, were built strictly in FORTRAN code. After years of use, with the coders since long removed, the ability to modify the existing code is limited. Moving forward into the 90 's, these same models can be updated and improved with the use of simulation languages. Simulation languages, such as Pritsker's SLAMSYSTEM, will provide extremely flexible and highly accurate analysis to reliability models.

The primary purpose for the creation of CVRASM is to observe the behavior of a combat vehicle as it operates in a particular environment. The environment is depicted in three phases as illustrated in figure $1 ;(1)$ "move to combat", (2) "combat operations", and (3) "scheduled maintenance". The parameters of these three phases, including the time length of each phase as well as the mean time to failure, (MFAIL), for each phase are user defined and inserted into the input file. Repair is accomplished on "failed" vehicles through the 


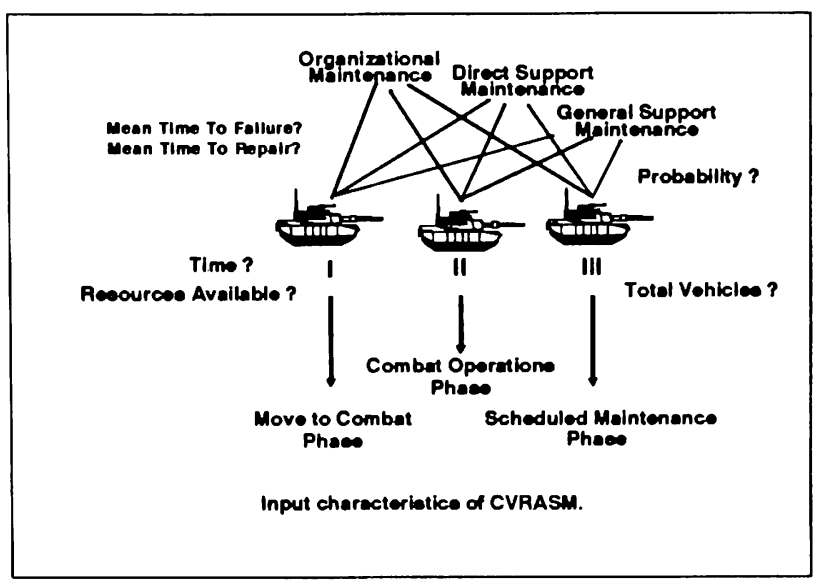

Figure 1: Characteristics of CVRASM

support teams located at the organizational, direct support, and general support maintenance facilities. To repair a tank which has failed the user inserts the probabilities associated with the requirement for each of these maintenance organizations, as well as the mean time to repair, (MREPAIR), at each level. The above mentioned parameters may be significantly different for the three previously discussed phases. For example, in the "combat" phase regular maintenance may not be performed adequately and the failure rate may occur more frequently than the "move to combat" phase. Further, the probability associated with the need of each maintenance level may also change significantly during each phase.

Additional consideration is in the allocation of resources at the three levels of maintenance. CVRASM allows the user to input the availability of assets, (servers), at each level to retrieve and service the vehicles.

Additional input variables defined by the user include the number of vehicles to be analyzed as well as the total simulation time. The example in section 5 lists the input variables. CVRASM analyzes the mechanical attrition and associated logistics aspects of a vehicle as it operates and is supported by maintenance teams for a defined scenario.

The model is written in Simulation Language for Alternative Modeling, (SLAM II), courtesy of Pritsker Corporation. The entire model runs under SLAMSYSTEM 2.1 providing total graphical user interface, GUI, which greatly enhances the output results. Such results are in the form of bar charts, plots, histograms, pie charts, written reports, and a visual animation. The subroutines are written in Microsoft FORTRAN Version 5.0.

\section{OBJECTIVES}

First: Develop a simulation model which can be used in (1) determining the reliability, availability, and maintainability, RAM, requirements, for new systems, and (2) evaluating the effects of various changes in support policy and logistics concepts on operational readiness.

Second: Promote the future development of simulations to be used at the lowest level of analysis so that immediate decisions can be employed at the user level. This PC-based simulation allows analysis to be accomplished at the lowest echelon without the burden of large mainframe computers.

Third: Promote the further use of simulation languages in the replacement of the "old" FORTRAN based systems. Such development will allow rapid change within the simulation to adapt to changing situations. Easily made changes within the graphical user interface, GUI, will provide instantaneous results at a greatly reduced programming cost.

\section{THE RELIABILITY MODEL}

\subsection{Model Background}

Reliability, $R(t)$, is the probability that a device, system, subsystem, or component, performs adequately over some time interval $[0, t]$. Consider the M1A1 main battle tank as an example. There is a very large number of parts, assemblies, and subsystems which comprise the total system or tank. Possible combined subsystems are critical to the operation of the tank itself and if inoperable may in fact "dead line" the vehicle from its primary function as a combat vehicle.

Critical components, such as those mentioned above, may be considered to be subsystems in series. Any one of these subsystem failures will "dead line" the entire system until repairs are administered. In other words, the vehicle cannot perform its primary function if the engine, transmission, main gun, etc., is inoperative. Thus a system configured in series has a time to failure, $t_{\text {fail }}$, given by the function;

$$
t_{\text {fail }}=\min \left\{t_{f 1}, t_{f 2}, t_{f 3}, \ldots, t_{f n}\right\} .
$$

Further, for a system in series, the reliability is given as;

$$
R\left(p_{1}, p_{2}, p_{3}, \ldots, p_{n}\right)=p_{1}^{*} p_{2}^{*} p_{3} \ldots p_{n}
$$


As an example, if the probability of the M1A1 transmission performing satisfactorily is .95 , and the engine is .88 , then the reliability of this series subsystem is given by

$$
\mathrm{R}=(.95)(.88)=83.6 \%
$$

On the other hand some components of the M1A1 tank will not cause the entire system to fail. For example, if the main turret motor should fail, the turret can still be moved manually. This is definitely not the preferred option, however, the system still remains effective. These subsystems are configured in parallel. In general, a system with ' $n$ ' components in parallel will fail only if all components fail. In other words, the system can perform if one of the ' $n$ ' components perform satisfactorily. This concept is referred to as redundancy because there are alternative components, working within the system, to continue the primary function. For a parallel system the time to failure is given by;

$$
\mathrm{t}_{\text {fail }}=\max \left\{\mathrm{t}_{\mathrm{f} 1}, \mathrm{t}_{\mathrm{f} 2}, \mathrm{t}_{\mathrm{f} 3}, \ldots \mathrm{t}_{\mathrm{fn}}\right\} .
$$

Further, for a parallel system, or subsystem, the reliability is given as;

$$
R\left(p_{1}, p_{2}, p_{3}, \ldots p_{n}\right)=1-\left(1-p_{1}\right)\left(1-p_{2}\right)\left(1-p_{3}\right) \ldots\left(1-p_{n}\right) .
$$

For example, revisiting the M1A1 example, if the probability that the gunner's fire control unit functions is .99 , and the commander's fire control unit is .95 , the fire control subsystem reliability is given by

$$
\mathrm{R}=1-(.01)(.05)=99.95 \% \text {. }
$$

We are concerned with performance as a function of time. Associated with the reliability of a component or subsystem is the random variable ' $\mathrm{T}$, called the time to failure. If we label ' $F$ the cumulative distribution function associated with ' $\mathrm{T}$ ' and ' $\mathrm{f}$ ' the density function for 'T then

$$
R(t)=1-F(t)=1-\int_{0}^{t} f(y) d y=\int_{t}^{\inf } f(y) d y .
$$

The failure rate, $r(t)$, is therefore $r(t)=f(t) / R(t)$. This implies that $r(t) d t$ represents the conditional probability that a component functioning properly at time ' $t$ ' will fail first in the interval $[t, t+d t]$. In many components the failure rate will increase because of normal wear and tear. This type of failure rate, either constant, or increasing with time, is known as increasing failure rate
(IFR). If the failure rate remains constant or decreases with time this concept is known as decreasing failure rate (DFR).

The exponential distribution is often used to model the time to failure of both DFR and IFR systems because as we see below the failure rate is constant. This comes about by the "memoryless" property of the exponential distribution. If the component failure distribution is exponential, then

$$
F(t)=1-e^{-t / \theta}
$$

Therefore $R(t)$ is given by $\mathrm{e}^{-t / \theta}$, and the failure rate is ,

$$
r(t)=\left[(1 / \theta) e^{-t / \theta}\right] / e^{-t / \theta}=1 / \theta
$$

Thus, the mean time to failure, MFAIL, and mean time to repair, MREPAIR, for this simulation model are the means of two exponential distributions.

In evaluating the random aspects of time to failure and time to repair the generation of random variates from the exponential cumulative distribution function is necessary. Further, it is necessary that a statistically reliable $\mathrm{U}(0,1)$, random-number generator be available. In this simulation the requirement is easily accomplished with SLAMSYSTEM. SLAMSYSTEM provides the random number generator and the inversetransformation for the exponential distribution. In essence through the inverse-transformation method SLAMSYSTEM accomplishes the following;

$$
\text { 1. Generate } R=U(0,1)
$$

$$
\text { 2. Return } X=F^{-1}(R)
$$

where ' $\mathrm{R}$ ' is the random number, and ' $\mathrm{X}$ ' is the random variate.

\subsection{Assumptions}

First: Each entity or M1A1 main battle tank, M2 Infantry Fighting Vehicle, etc., is analyzed as a subsystem of the combat unit. These subsystems operate in parallel, thus when another tank fails, total system operation can continue. In clear context, the other tanks continue to operate regardless if one or more of their compatriots is temporarily down for repair operations. This allows the model to assess reliability with repair operations for the number of tanks in operation and equipment recycling following repair activities. Total system failure occurs when the total number of vehicles "down" reaches a prescribed minimum. 
Second: Each entity has been previously studied to insure the input parameters for failure statistics as well as repair statistics have been verified. This information is obtainable from manufacturers' specifications or more accurately obtained from on-site historical maintenance documentation. This information is available from organizational maintenance (ORG), direct support maintenance (DS), and general support maintenance (GS) facilities. In the absence of such data, simulations should be used to evaluate these types of vehicles. These simulations should initially model the vehicles in series, and parallel configurations as described in paragraph 3.1.

Third: Goodness-of-fit distribution tests should be accomplished to verify the probability distributions of the failure and repair times. Such procedures most commonly include the chi-square and KolmogorovSmirnov tests.

Fourth: Time to repair at the organizational, direct support, and general support facilities are accurate and depict the scenario under study, including the time to locate the downed vehicle, time to make an assessment, towing operations, maintenance repair times, and return to the battle field.

Fifth: This simulation does not assess combat losses.

\subsection{The SLAM II Model}

The simulation language used to model this particular project was Simulation Language for Alternative Modeling II, SLAM II, courtesy Pritsker Corporation. Figure 2 provides a detailed drawing of the model. The model was developed under the SLAMSYSTEM 2.1 total project support package. The FORTRAN subroutines were developed with Microsoft FORTRAN Version 5.0.

\section{OUTPUT ANALYSIS}

\subsection{Input Report Identification}

The program designed to run under SLAMSYSTEM allows the user to create input data bases for vehicles under study. These data bases are labeled M1A1.DAT, M2.DAT, etc.. To activate the data base in question, the user merely makes the data base active at the opening menu.

Information entry is further simplified with the use of Microsoft Excel 3.0. This spreadsheet program uses the dynamic data exchange, DDE, capabilities to systematically enter the input variables. This system eleviates the user the burden of specialized input routines to properly track the data This becomes very important particularly when the number of variables becomes very large, i.e., into the hundreds, or even thousands.

During the execution of the simulation the user is asked to pick the data base to use for the simulation. This allows for easy contrast and comparisons of output results and affords the user the ability to perform sensitivity analysis on the appropriate variables.

The following input variables are preset in the model to adhere to a standard on which to base comparisons:

Preset Variables (inclusive of all three phases)

(1) Simulation Run Time .240 hours

(2) Histograms .240 hours

(3) Bar Charts

(4) Pie Charts

(5) Plots

(6) Output Summary Reports .every 24 hours

User Set Variables (inclusive of all three phases)

(1) Total Number of Vehicles

(2) Mean Time Between Failures

(3) Organizational Maintenance Mean Repair Time

(4) Direct Support Maintenance Mean Repair Time

(5) General Support Maintenance Mean Repair Time

(6) Probabilities of Occurrence of (3), (4), and (5)

(7) Commanders' Specified Combat Minimum

\subsection{Output Report Identification}

The output results produced by this model under the SLAMSYSTEM environment provides easily readable reports every 24 hours of operation inclusive of the 240th hour, and easily understood graphics to support the reports. Such graphics include:

\section{(1) Bar Charts}

a. Observed Mean Statistics to reference number of failures, repairs, and combat minimums in relation to time.

b. Queue statistics to reference operating characteristics such as average wait times, average queue lengths, total entity counts processed at each facility, and current utilization of each processing station. 


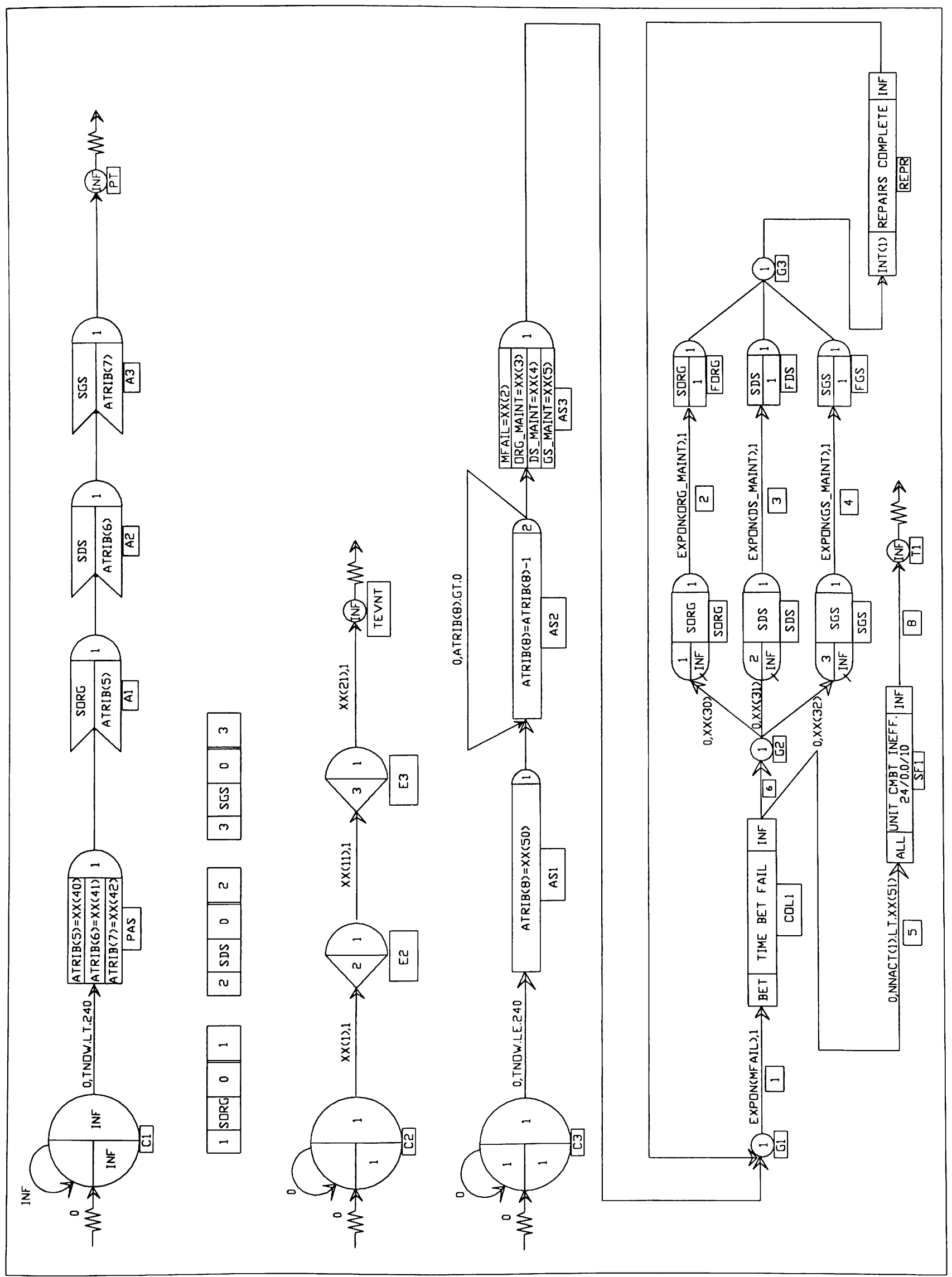

Figure 2: SLAM II Network Model of CVRASM 
(2) Pie Charts

Utilization statistics to reference availability of the various work stations, ie., organizational, direct support, and general support maintenance facilities.

\section{(3) Plots}

Provides accurate plots of the units vehicle availability over time. This provides the user with accurate information at any time period in the simulation to address the number of vehicles available during any of the three phases of operation. Additional plots are available to monitor the number of vehicles in the repair queues over time, cumulative repairs at each maintenance facility, and the total number of failures of the vehicle type under study.

\section{A NUMERICAL EXAMPLE}

\subsection{The Input Parameters}

The table below represents sample data initially "run" in this simulation model.

\begin{tabular}{|l|c|c|c|}
\hline & $\begin{array}{c}\text { Phase } \\
1\end{array}$ & $\begin{array}{c}\text { Phase } \\
2\end{array}$ & $\begin{array}{c}\text { Phase } \\
3\end{array}$ \\
\hline Time Increment & $72.00 \mathrm{~h}$ & $144.0 \mathrm{~h}$ & $24.00 \mathrm{~h}$ \\
\hline Mean Time To Failure & $42.90 \mathrm{~h}$ & $35.70 \mathrm{~h}$ & $41.40 \mathrm{~h}$ \\
\hline ORG Mean Repair & $1.70 \mathrm{~h}$ & $4.67 \mathrm{~h}$ & $2.20 \mathrm{~h}$ \\
\hline DS Mean Repair & $3.70 \mathrm{~h}$ & $12.56 \mathrm{~h}$ & $4.80 \mathrm{~h}$ \\
\hline GS Mean Repair & $7.60 \mathrm{~h}$ & $36.78 \mathrm{~h}$ & $32.47 \mathrm{~h}$ \\
\hline Probability ORG Maint & .77 & .82 & .60 \\
\hline Probability DS Maint & .20 & .10 & .25 \\
\hline Probability GS Maint & .03 & .08 & .15 \\
\hline ORG Service Teams & 2 & 2 & 2 \\
\hline DS Service Teams & 2 & 2 & 2 \\
\hline GS Service Teams & 2 & 2 & 2 \\
\hline Total Vehicles & 58 & 58 & 58 \\
\hline Combat Minimum & 45 & 45 & 45 \\
\hline
\end{tabular}

The matrix above represents the initial data for the three phases of combat. For example, the Phase 1 operation, "Move To Combat", depicts a 72 hour duration. The time to failure for the tank or system is exponential with a mean of 42.90 hours. The three sources of repair; organization, direct support, and general support, have repair times exponential with mean times of $1.70,3.70$, and 7.60 hours respectively. The probability of each of the "down" vehicles needing repair at these three repair facilities is $.77, .20$, and .03 . There are currently 2 service teams available at each of these stations. The initial starting level of these vehicles is a total of 58 tanks. The commander has requested a "flag" be inserted to notify him of the time periods the unit will fall below the combat minimum available which is set at 45 vehicles. The other two phases, "Combat Operations", and "Scheduled Maintenance" function similarly. The simulation will automatically change these phase variables as the simulation time progresses.

\subsection{Output Results}

Figures 3, 4, 5, and 6 represent the "most important" information from the SLAMSYSTEM output data summary report as well as the output graphical files.

Figure 3 provides information pertaining to the number of M1A1 tanks available over the 240 hour time sequence. This information is valuable particularly in determining the optimized mixture of the service facilities to support the total operation to the commander's criteria.

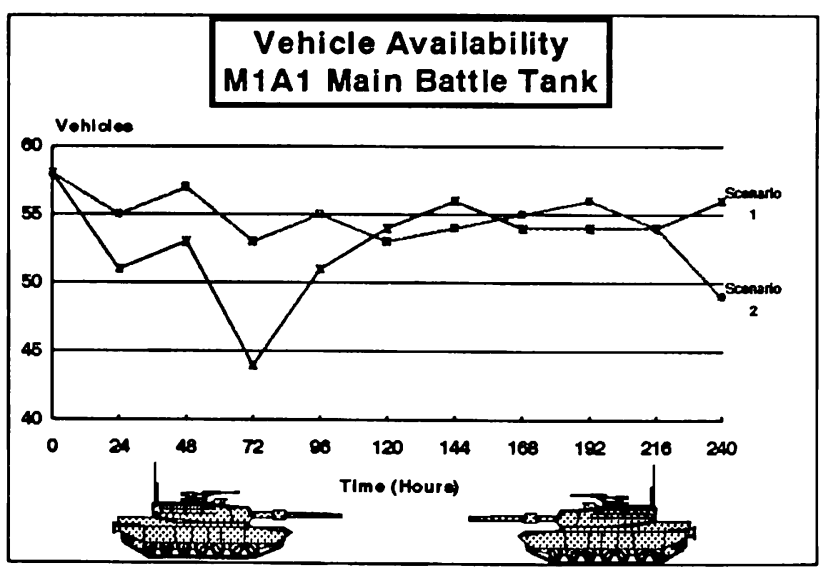

Figure 3: Vehicle Availability VS Time

Optimization is accomplished through the coordinate search or ceteris paribus approach. The procedure is to identify those variables which the commander can easily alter or control, such as the number of servers available. These independent variables are labeled $x_{1}, x_{2}, \ldots$ $\mathrm{x}_{\mathrm{n}}$. Next, we change one independent variable at a time until no improvement results. Then, we change a second variable while keeping the first variable at its apparent optimal setting, and continue this procedure until all variables are at their optimum level. This method is also depicted in figure 3. Two simulations are represented. Scenario 1 represents our original data with two service facilities at the organizational level. Scenario 2 represents moving one team from the general support level to the organizational level, thus 
allocating three service teams to organizational maintenance and reducing the service level of the general support maintenance to one team. The improvement of this variable change is significant. The number of vehicles available throughout the simulation is much higher as seen in scenario 2.

Figure 4 depicts service completions for the three service facilities. This graph reveals that the organizational maintenance performed 231 repair operations and had $(0)$ in progress at the termination of the simulation. The direct support and general support maintenance facilities are similarly represented.

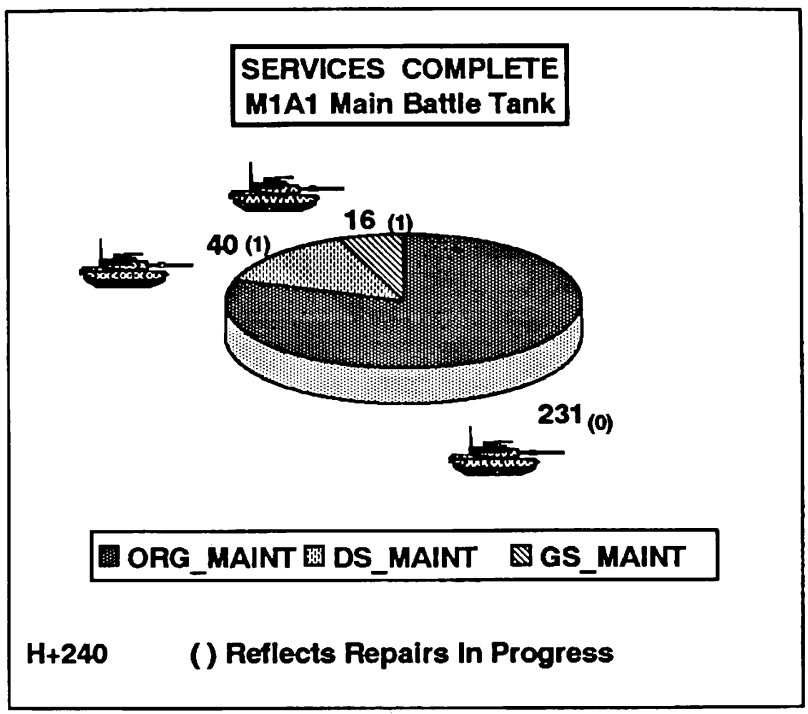

Figure 4: Vehicle Services Completed

Figure 5 provides another aspect of server utilization; percent busy and idle. Accumulated statistics over a 240 hour period reflects that the organizational maintenance was busy $84 \%$ of the period and idle $16 \%$ of the period. The direct support and general support facilities are also shown for comparison. This utilization graph is important reflecting the changes in work load which provide another critical factor which the commander needs to consider, particularly in extended operations where the maintenance crews may not be able to sustain at high levels of utilization for long durations.

The histogram, seen in figure 6 , provides valuable information to the commander in regards to his unit's combat ineffectiveness. Remembering the commander's criteria of sustaining a combat minimum of 45 vehicles, results reveal that during the 70 through 90 hour time frame the unit became combat ineffective. Specifically, during the 70 to 80 hour period the unit fell five units below the commander's minimum. Therefore, the total vehicles available was 40 . Similarly during the 80 to 90 hour time period the unit fell below the commander's

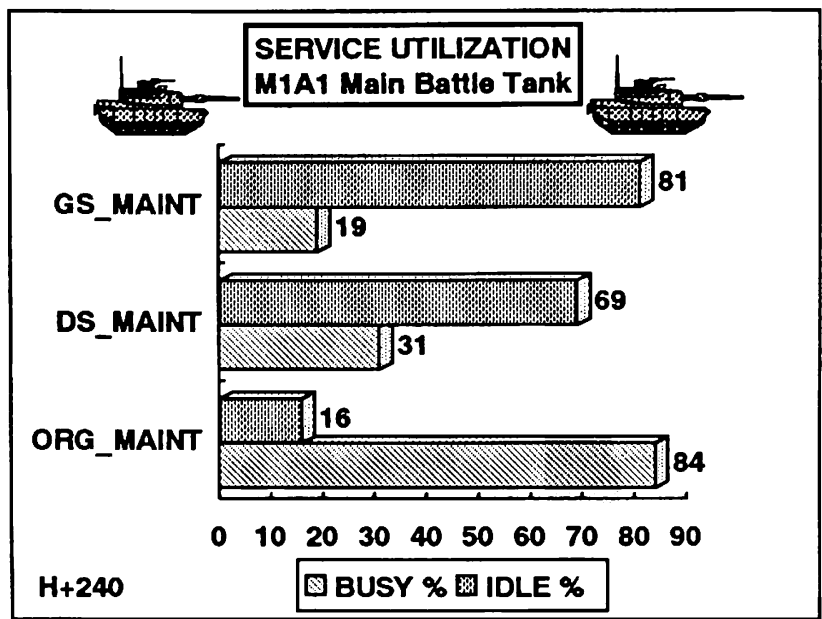

Figure 5: Service Utilization

minimum by two vehicles, leaving only 43 vehicles available. This information allows the commander to analyze the situation and optimize his maintenance configuration in order to insure that the highest possible amount of vehicles are combat ready.

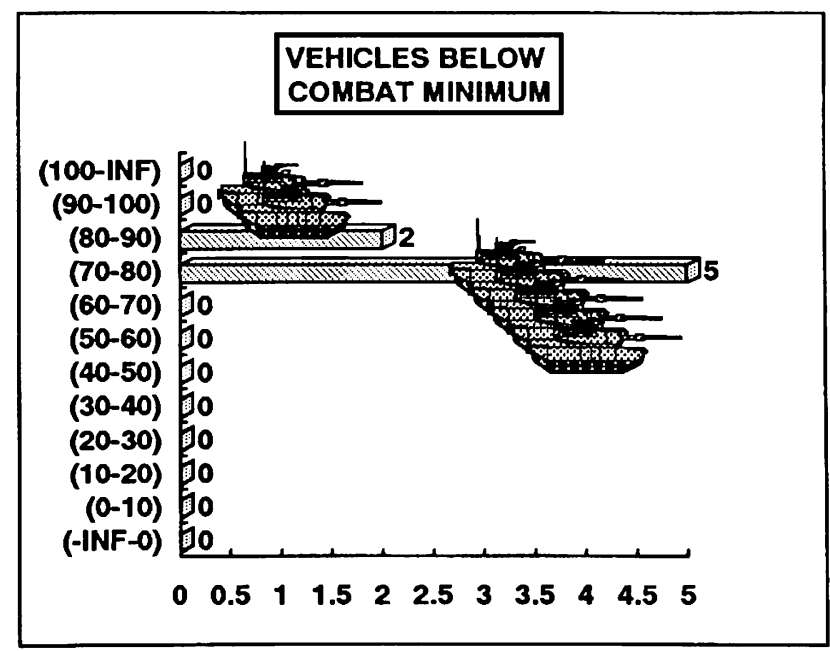

Figure 6: Histogram of Vehicles Below Combat Minimum

\section{BENCHMARK RESULTS}

The simulation developed in this paper was run on a IBM PS/2 Model 70; type 121; 20 MHZ; 6 megabytes of RAM; and a 387 math coprocessor. Total run time for the simulation was 1.5 minutes with an additional 30 seconds for graphical output reporting. The average output in summary format was 61 pages per simulation run. Each report represents a total of ten reports providing the same output as described above. 


\section{VERIFICATION AND VALIDATION}

Verification was performed by an analysis of the output data. All aspects of the simulation were tested, including pushing the minimum and maximum limits of each variable. Calculations of the means, standard deviations, coefficients of variation, absolute minimum values, absolute maximum values, and queue operating characteristics were checked. The model performs as intended.

Validation is more difficult to achieve. Validation, or testing and checking all known assumptions, and deficiencies of the model is ongoing. It is hopeful that using agencies will test and further refine this model. Additionally, the model is supported with an animation, contributing to the validation process.

\section{CONCLUDING REMARKS}

A simulation model to analyze the reliability claims of manufacturers in conjunction with combat operations has been developed. Further, this model allows the user to manipulate current repair policies and procedures to maximize the availability of the combat vehicles. Such results will adequately analyze the status of equipment over long time periods and provide an initial basis to predict the number of systems that are necessary to perform a specified mission. Additionally, this allows the testing agency to analyze the actual performance of the vehicles or equipment and verify the manufacturers specifications. Further, this analysis will provide major theater commanders with a logical understanding of the repair facilities needed to insure that combat minimums are maintained.

This model is also applicable to the civilian market as a means to analyze systems, such as generators, trucks, automobiles, manufacturing equipment, etc. Using this model in conjunction with cost analysis provides a basis for total repair cost estimates.

Every attempt has been made to insure that this model represents the system under observation. Secondly, great consideration is taken to insure that the model is timely and extendible, and third that the model is understandable, and user-friendly.

\section{DISCLAIMER}

The views, analyses, and findings in this report represent the views of the author, and do not represent the official position of the United States Army or the Department of Defense.

\section{ACKNOWLEDGMENTS}

The author wishes to thank LTC Lee Dewald, Ph.D, and the staff from the U.S. Army Logistics Management College, Fort Lee, Virginia; Mr. Bob Morrison, Combat Support Command; Ms. Jean O'Reilly, Kenneth Musselman, Ph.D, and Ms. Jenny Mischler, from PRITSKER CORPORATION, 1305 Cumberland Avenue, West Lafayette, Indiana, for their continual support in my research in simulation.

\section{REFERENCES}

Bishop, C., and I. Drury. 1988. The Encyclopedia of World Military Weapons. London, England: Aerospace Publishing Ltd.

Hillier, F.S., and G.J. Lieberman. 1986. Introduction To Operations Research, Fourth Edition. Oakland: Holden-Day.

Kleijnen, J.P. 1987. Statistical Tools For Simulation Practitioners. New York: Dekkar and Inc.

Law, A.M., and W.D. Kelton. 1991. Simulation Modeling And Analysis, Second Edition. New York: McGraw-Hill.

Milton, J.S., and J.C. Arnold. 1986. Probability And Statistics In The Engineering And Computing Sciences. New York: McGraw-Hill.

Pritsker, A.A.B. 1986. Introduction To Simulation and SLAM II, Third Edition. New York: Halsted Press. Pritsker, A.A.B., E. Sigal, and J. Hammesfahr. 1989. SLAM II Network Models For Decision Support.New Jersey: Prentice-Hall.

Pritsker, A.A.B. 1990. Papers Experiences Perspectives. West Lafayette: Systems Publishing Corporation.

Pritsker, A.A.B. 1989.Modeling Viewpoints For Assessing Reliability. West Lafayette: Systems Publishing Corporation.

Taha, H.A. 1987. Operations Research An Introduction, Fourth Edition. New York: Macmillan, Inc.

\section{AUTHOR BIOGRAPHY}

STEPHEN R. PARKER is an operations research instructor assigned to the Department of Decision Sciences at the U.S. Army Logistics Management College, Fort Lee, Virginia. He is a senior member of the Institute of Industrial Engineers, and certified as a Professional Engineer in the state of Virginia. He is a Major in the United States Army. His research interest is in stochastic simulations and optimization. 